\title{
Productivity improvement with parallel adjacent U-shaped assembly lines
}

\author{
Chutima, P. ${ }^{\mathrm{a}, \mathrm{b},{ }^{*}}$, Suchanun, T. ${ }^{\mathrm{a}}$ \\ ${ }^{a}$ Industrial Engineering, Faculty of Engineering, Chulalongkorn University, Bangkok, Thailand \\ ${ }^{b}$ Academy of Science, The Royal Society of Thailand, Bangkok, Thailand
}

\begin{abstract}
A B S T R A C T
A novel configuration of assembly lines was proposed in this research, namely parallel adjacent U-shaped assembly lines (PAUL). Typically, in a multiple Uline facility, each U-line is designed to work independently which may cause some workstations were not fully functioned. The PAUL aimed at increasing the utilisation of the whole facility by allowing cross-trained workers to work on the opposite legs of the adjacent U-lines (multi-line workstations). This configuration is easier to implement than parallel U-lines due to no restriction in terms of the lengths of U-lines to be paralleled and hidden expenditures that could be incurred in shop floor reconstruction. Since the line balancing of the PAUL is NP-hard and many conflicting objectives need to be optimised simultaneously, the evolutionary meta-heuristic which was the hybridisation of the multi-objective evolutionary algorithm based on decomposition (MOEA/D) and particle swarm optimisation (PSO), namely MOEA/D-PSO, was developed to effectively solve the problem. In addition, the decoding algorithm to convert the solutions obtained from MOEA/D-PSO into the PAUL's configuration was proposed. The performance of MOEA/D-PSO was evaluated against MOEA/D and multi-objective particle swarm optimisation (MOPSO). The experimental results reveal that MOEA/D-PSO outperformed its rival algorithms under the convergence-related performance.
\end{abstract}

\author{
ARTICLE INFO \\ Keywords: \\ Assembly line; \\ U-shaped assembly line; \\ Parallel adjacent assembly line; \\ Assembly line balancing; \\ Productivity improvement; \\ Multi-objective optimisation; \\ Evolutionary algorithm (MOEA/D); \\ Particle swarm optimisation (PSO) \\ *Corresponding author: \\ cparames@chula.ac.th \\ (Chutima, P.)
}

Article history:

Received 29 October 2018

Revised 20 December 2019

Accepted 21 January 2019 (C) 2019 CPE, University of Maribor. All rights reserved.

\section{References}

[1] Cheng, C.H., Miltenburg, J., Motwani, J. (2000). The effect of straight- and U-shaped lines on quality, IEEE Transactions on Engineering Management, Vol. 47, No. 3, 321-334, doi: 10.1109/17.865901.

[2] Gökçen, H., Ağpak, K., Benzer, R. (2006). Balancing of parallel assembly lines, International Journal of Production Economics, Vol. 103, No. 2, 600-609, doi: 10.1016/j.ijpe.2005.12.001.

[3] Küçükkoç, I., Zhang, D.Z. (2015). Balancing of parallel U-shaped assembly lines, Computers \& Operations Research, Vol. 64, 233-244, doi: 10.1016/j.cor.2015.05.014.

[4] Chutima, P., Chimklai, P. (2012). Multi-objective two-sided mixed-model assembly line balancing using particle swarm optimisation with negative knowledge, Computers \& Industrial Engineering, Vol. 62, No. 1, 39-55, doi: 10.1016/j.cie.2011.08.015.

[5] Zhang, Q., Li, H. (2007). MOEA/D: A multiobjective evolutionary algorithm based on decomposition, IEEE Transactions on Evolutionary Computation, Vol. 11, No. 6, 712-731, doi: 10.1109/TEVC.2007.892759.

[6] Hassan, R., Cohanim, B., de Weck, O., Venter, G. (2005). A comparison of particle swarm optimisation and the genetic algorithm, In: Proceedings of 46th AIAA/ASME/ASCE/AHS/ASC Structures, Structural Dynamics and Materials Conference, Austin, Texas, USA, doi: 10.2514/6.2005-1897.

[7] Kennedy, J., Eberhart, R. (1995). Particle swarm optimization, In: Proceedings of ICNN'95 - International Conference on Neural Networks, Perth, WA, Australia, Vol. 4, 1942-1945, doi: 10.1109/ICNN.1995.488968.

[8] Coello, C.A.C., Cortés, N.C. (2005). Solving multiobjective optimisation problems using an artificial immune system, Genetic Programming and Evolvable Machines, Vol. 6, No. 2, 163-190, doi: 10.1007/s10710-005-6164-x. 
[9] Chutima, P., Olarnviwatchai, S. (2016). A multi-objective car sequencing problem on two-sided assembly lines, Journal of Intelligent Manufacturing, Vol. 29, No. 7, 1617-1636, doi: 10.1007/s10845-016-1201-6.

[10] Corominas, A., Pastor, R., Plans, J. (2008). Balancing assembly line with skilled and unskilled workers, Omega, Vol. 36, No. 6, 1126-1132, doi: 10.1016/j.omega.2006.03.003.

[11] Tiacci, L. (2017). Mixed-model U-shaped assembly lines: Balancing and comparing with straight lines with buffers and parallel workstations, Journal of Manufacturing Systems, Vol. 45, 286-305, doi: 10.1016/i.jmsy.2017.07. $\underline{005}$.

[12] Defersha, F.M., Mohebalizadehgashti, F. (2018). Simultaneous balancing, sequencing, and workstation planning for a mixed model manual assembly line using hybrid genetic algorithm, Computers \& Industrial Engineering, Vol. 119, 370-387, doi: 10.1016/i.cie.2018.04.014. 


\title{
Izboljšanje produktivnosti z vzporednimi sosednjimi montažnimi linijami v obliki črke $U$
}

\author{
Chutima, P. ${ }^{\mathrm{a}, \mathrm{b},{ }^{*}}$, Suchanun, T. $^{\mathrm{a}}$ \\ andustrial Engineering, Faculty of Engineering, Chulalongkorn University, Bangkok, Thailand \\ ${ }^{b}$ Academy of Science, The Royal Society of Thailand, Bangkok, Thailand
}

\section{POVZETEK}

$\mathrm{V}$ tej raziskavi je bila predlagana nova konfiguracija montažnih linij, in sicer vzporednih sosednjih montažnih linij v obliki črke U (PAUL). Običajno je v objektih s postavitvijo več U-linij vsaka U-linija zasnovana tako, da deluje neodvisno, kar lahko povzroči, da nekatere delovne postaje niso bile popolnoma delujoče. Cilj konfiguracije PAUL je bil povečati izkoristek celotnega objekta, tako da je usposobljenim delavcem omogočil delo na nasprotnih krakih sosednjih U-linij (več linijske delovne postaje). To konfiguracijo je lažje izvesti kot vzporedne U-linije, ker ni omejitev glede dolžine vzporednih U-linij in skritih stroškov, ki bi lahko nastali pri preoblikovanju proizvodne hale. Ker je uravnoteženje linij PAUL računsko zahtevno in je treba hkrati optimizirati številne nasprotujoče si kriterije, je bil razvit evolucijski metahevristični algoritem MOEA/D-PSO, ki je hibridizacija večkriterijskega evolucijskega algoritma, ki temelji na razgradnji (MOEA/D) in optimizaciji z rojem delcev (PSO). Poleg tega je bil predlagan algoritem za pretvorbo rešitev, pridobljenih iz MOEA/D-PSO, v konfiguracijo PAUL. Učinkovitost MOEA/D-PSO je bila ovrednotena glede na MOEA/D in glede na večkriterijsko optimizacijo z rojem delcev (MOPSO). Eksperimentalni rezultati razkrivajo, da je MOEA/D-PSO v konvergenčni uspešnosti presegel konkurenčne algoritme.

\section{PODATKI O ČLANKU}

Ključne besede:

Montažna linija;

Montažna linija v obliki črke U;

Vzporedno sosednja montažna linija;

Uravnoteženje montažne linije; Izboljšanje produktivnosti;

Večkriterijska optimizacija;

Evolucijski algoritem (MOEA/D) ;

Optimizacija z rojem delcev (PSO)

*Kontaktna oseba:

cparames@chula.ac.th

(Chutima, P.)

Zgodovina članka:

Prejet 29. oktobra 2018

Popravljen 20. decembra 2018

Sprejet 21. januarja 2019 